\title{
US right to try laws and BioPharma
}

Volume 2 Issue 4 - 2015

\section{Keywords: clinical trials, BioPharma, right to try, FDA}

\section{Commentary}

A few years ago in a graduate course I coordinate on drug discovery issues, one of my guest lecturers on the topic of state government affairs and their impact on the BioPharma industry commented that frequently the states are referred to as the "laboratories of democracy." From the standpoint of the states experimenting with policy issues prior to the Federal Government he is correct for oftentimes the states are the birthplace of Federal Policy: Welfare Reform (Wisconsin); Medicaid Best Price (California); and Health Insurance Reform (Massachusetts). Indeed the labs have been very busy with over 7,300 bills being tracked (Bill Track 50) in 2014 that have health care in their titles. But perhaps one of the most sensitive areas of legislation being pursued at state levels is what is termed "right to try" laws.

These laws have passed rather quickly in Colorado, Michigan, Missouri, Louisiana and Arizona with legislators in other states promising to introduce similar bills in 2015. Basically what these bills in one form or another propose to do is to give seriously or critically ill patients the right to access medications that have not been approved by the FDA and have only been through Phase I clinical trials. While there are slight variations from state-to-state, common language amongst the bills stipulates that once a patient has exhausted all other therapeutic options, the patient and his/her doctor can approach a BioPharma firm for permission to gain access to a drug even though it has only been through Phase I clinical trials. The major operational and ethical caveat in all this is that Phase I clinical studies only involve perhaps $20-50$ patients, usually normal healthy volunteers, and is really focused on determining if the potential therapeutic is safe and tolerated: Not does it cure or treat a disease. In essence, these laws aim to hasten access to hopefully lifesaving but unapproved drugs by completely removing oversight by the FDA. This would all be a good for society and terminally ill patients if the driving force of increased accessibility was a real problem but it isn't. The FDA already has in place an "expanded access" or "compassionate use" program for granting access to unapproved therapies in patients with exceptional medical needs.

A spokesperson for the FDA has noted that they allow about 1,000 patients access to unapproved therapeutics via this mechanism each year. Recently the FDA, taking to heart the criticism that the online form was too long, has significantly shortened the form thus simplifying the process even more. The positive aspect of this is that even though "compassionate use" protocols permit usage outside of the standard FDA clinical trial schema, the FDA still has oversight of the process. However, organizations such as the Goldwater Institute and legislators pushing these bills feel that the FDA clinical trial process is archaic and a "compassionate use" request a bureaucratic waste of time limiting patient access to lifesaving medications. From a business standpoint, these "right to try" laws present significant risk to both small and large firms especially in the growing field of immunooncology or other potential niche products. Some of the provisions of

\author{
Dennis M Gross \\ Pennsylvania Drug Discovery Institute, USA
}

Correspondence: Dennis M Gross, Pennsylvania Drug Discovery Institute, Pennsylvania Biotechnology Center, 3805 Old Easton Road, Doylestown, PA I8902, USA, Tel I2I59398966, Email dgross@padrugdiscovery.org

Received: July 31, 2015 | Published: October 06, 2015 these bills state that the drug's manufacturer can, but is not required to make the drug available, and can choose whether or not to charge the patient. Furthermore, while state medical board disciplinary action against the doctor because of untoward patient complications might be blocked by the new legislation, both the doctor and the manufacturer would remain subject to liability for injuries to the patient even if both comply with the tenets of the act. Making this even more bizarre is that any state employee, official or agent of a state who blocks a patient's access or even attempts to block access via this new legislation is guilty of a misdemeanor subject to prison time and a fine.

More fundamentally however are the ethical issues this legislation brings to the forefront. In the view of Dr. Arthur Caplan, a bioethicist at NYU Langone Medical Center, "These laws are easy to vote for but accomplish almost nothing". They're feel-good laws. Therein lies the conundrum for BioPharma. These state laws won't force a firm to make their drug available with only minimal Phase I safety data but it will put them in the public spotlight having refused to do so. It forces them to subsequently bypass the FDA regulatory process they committed themselves to so they can obtain data to determine if their drug actually works in the target population. Even permitting a drug undergoing clinical trials to be used under a FDA "compassionate use" protocol still provides FDA oversight and supervision and insures the collection of robust data that can ultimately compliment the data from the full clinical trials. As Dr. Ezakiel Emanuel of the University of Pennsylvania noted, "You don't learn anything useful from the one-off cases." but failure will most assuredly create barriers to entry with the FDA, the private funding community and the physicians who will be making decisions of usage based upon scientific principles not patient anecdotes. As Jann Bellamy noted last year, this is "Public theater at taxpayer expense" or as my guest lecturer also noted, perhaps we should take away some of the test tubes from the "labs of democracy."

\section{Acknowledgements}

None. 


\section{Conflicts on interest}

The author declares that there is no conflict of interest.

\section{Funding}

None. 